\title{
Speaker and Digit Recognition by Audio-Visual Lip Biometrics
}

\author{
Maycel Isaac Faraj and Josef Bigun \\ Halmstad University, School of Information Science, \\ Computer and Electrical Engineering (IDE) \\ Halmstad University, Box 823, SE-301 18 Halmstad \\ \{maycel.faraj, josef.bigun\}@ide.hh.se
}

\begin{abstract}
This paper proposes a new robust bi-modal audio visual digit and speaker recognition system by lip-motion and speech biometrics. To increase the robustness of digit and speaker recognition, we have proposed a method using speaker lip motion information extracted from video sequences with low resolution $(128 \times 128$ pixels $)$. In this paper we investigate a biometric system for digit recognition and speaker identification based using line-motion estimation with speech information and Support Vector Machines. The acoustic and visual features are fused at the feature level showing favourable results with digit recognition being $83 \%$ to $100 \%$ and speaker recognition $100 \%$ on the XM2VTS database.
\end{abstract}

\section{Introduction}

In recent years, some techniques have been suggested that combine visual features to improve the recognition rate in acoustically noisy environments that have background noise or cross talk among speakers [1] 2, 3] [4] [5]. The present work is a continuation of [6]. The dynamic visual features are suggested based on the shape and intensity of the lip region [7] [8] [9] [10] because changes in the mouth shape including the lips and tongue carry significant phoneme-discrimination information. So far the visual representation has been based on shape models to represent changed mouth shapes that rely exclusively on the accurate detection of the lip contours, often a challenging task under varying illumination conditions and rotations of the face. Another disadvantage is the fluctuating computation time due to the iterative convergence process of the contour extraction. The motion in dynamic lip images can be modelled by moving-line patterns generating planes in space-time that encode the normal velocity of lines also known as normal image velocity, further details can be read in [6] [12].

Here we use direct feature fusion to obtain the audio-visual observation vectors by concatenating the audio and visual features. The observation sequences are then modelled with a Support Vector Machine (SVM) classifier for digit and speaker recognition respectively. The studies [13] 14] 15] reported good performance with Support Vector Machine (SVMs) classifiers in recognition, whereas traditional methods for speaker recognition are GMMs 16 and artificial neural networks [17. By investigating SVM instead of the more common GMM [6], 
we wanted to study the performance influence of the classification method on speaker recognition and digit recognition.

In previous work [6], we introduced a novel feature extraction method for lip motion used in a speaker verification system as a framework for the well known Gaussian Mixture Models. Here, we extended previous work 6] by studying a novel quantization technique for lip features. Furthermore, a digit recognition is presented together with a biometric speaker identification using SVM classifier. An extension of this work as journal article is under review IEEET.onComputers. The remainder of the paper is organized as follows. In Section 2 we describe briefly the lip-motion technique for the mouth region along with our quantization (feature-reduction) method, followed by acoustic feature extraction in Section 3 . Section 4 describes SVM classifier used for digit and speaker recognition along with the database and the experimental setup in Section 5 . Finally experimental results are shown with a discussion of the experiments and the remaining issues, Section 6 and 7 .

\section{Visual Features by Normal Image Velocity}

Bigun et al. proposed a different motion estimation technique based on an eigenvalue analysis of the multidimensional structure tensor [18, allowing the minimization process of fitting a line or a plane to be carried without the Fourier Transform. Applied to optical-flow estimation, known as the 3D structure-tensor method, the eigenvector belonging to the largest eigenvalue of the tensor is directed in the direction of the contour motion, if motion is present. However, this method can be excessive for applications that need only line-motion features. We assume that the local neighbourhood in the lip image contains parallel lines or edges as this is supported by real data 19. Lines in a spatio-temporal image translated with a certain velocity in the normal direction will generate planes with a normal that can be estimated in a total-least-square-error (TLS) sense as the local directions of the lines in 2D manifolds using complex arithmetic and convolution [18. The velocity component of translation parallel to the line cannot be calculated; this is referred to as the aperture problem. We denote the normal unit vector as $\mathbf{k}=\left(k_{x}, k_{y}, k_{t}\right)^{T}$ and the projection of $\mathbf{k}$ to the $x-y$ coordinate axes represents the direction vector of the line's motion. The normal, $\mathbf{k}$, of the plane will then relate to the velocity vector $v \mathbf{a}$ as follows

$$
\begin{aligned}
\mathbf{v}=v \mathbf{a}=-\frac{k_{t}}{k_{x}^{2}+k_{y}^{2}}\left(k_{x}, k_{y}\right)^{T}= & \\
& -\frac{1}{\left(\frac{k_{x}}{k_{t}}\right)^{2}+\left(\frac{k_{y}}{k_{t}}\right)^{2}}\left(\frac{k_{x}}{k_{t}}, \frac{k_{y}}{k_{t}}\right)^{T},
\end{aligned}
$$

where $\mathbf{v}$ is the normal image flow. The normal velocity estimation problem becomes a problem of solving the tilts $\left(\tan \gamma_{1}=\frac{k_{x}}{k_{t}}\right)$ and $\left(\tan \gamma_{2}=\frac{k_{y}}{k_{t}}\right)$ of the motion plane in the $x t$ and $y t$ manifolds, which is obtained from the eigenvalue analysis of the $2 \mathrm{D}$ structure tensor, [18. Using complex numbers and smoothing, the angles 


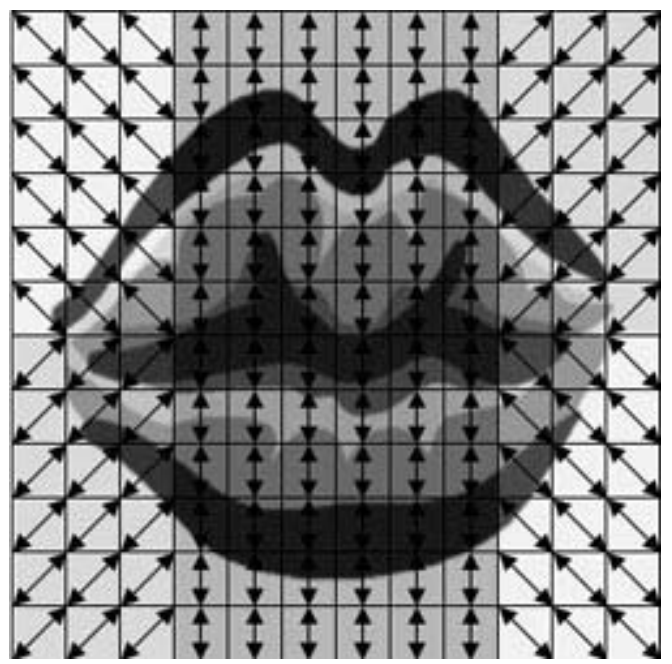

Fig. 1. Illustration of velocity estimation quantification and reduction

of the eigenvectors are given effectively as complex values such that its magnitude is the difference of the eigenvalues of the local structure tensor in the $x t$ manifold, whereas its argument is twice the angle of the most significant eigenvector approximating $2 \gamma_{1}$. The function $f$ represents the continuous local image, whose sampled version can be obtained from the observed image sequence. Thus, the arguments of $\tilde{u}_{1}$ and $\tilde{u}_{2}$ deliver the TLS estimations of $\gamma_{1}$ and $\gamma_{2}$ in the local 2D manifolds $x t$ and $y t$ respectively, but in the double angle representation [20, leading to the estimated velocity components as follows.

$$
\begin{aligned}
& \frac{k_{x}}{k_{t}}=\tan \gamma_{1}=\tan \left(\frac{1}{2} \arg \left(\tilde{u}_{1}\right)\right) \Rightarrow \tilde{v}_{x}=\frac{\tan \gamma_{1}}{\tan ^{2} \gamma_{1}+\tan ^{2} \gamma_{2}} \\
& \frac{k_{y}}{k_{t}}=\tan \gamma_{2}=\tan \left(\frac{1}{2} \arg \left(\tilde{u}_{2}\right)\right) \Rightarrow \tilde{v}_{y}=\frac{\tan \gamma_{2}}{\tan ^{2} \gamma_{1}+\tan ^{2} \gamma_{2}}
\end{aligned}
$$

The tilde over $v_{x}$ and $v_{y}$ denote that these quantities are estimations of $v_{x}$ and $v_{y}$. With the calculated 2D-velocity feature vectors, $\left(v_{x}, v_{y}\right)^{T}$, in each mouth-region frame $(128 \times 128$ pixels $)$ we have dense $2 \mathrm{D}$-velocity vectors. To extract statistical features from the $2 \mathrm{D}$ normal velocity and to reduce the amount of data without degrading identity-specific information excessively, we reduce the $2 \mathrm{D}$ velocity feature vectors $\left(v_{x}, v_{y}\right)^{T}$ at each pixel to $1 \mathrm{D}$ scalars where the expected directions of motion are $0^{\circ}, 45^{\circ},-45^{\circ}$ - marked with 3 different greyscale shades in 6 regions in Fig. 1. The motion vectors within each region become real scalars that take the signs + or - depending on which direction they move relative to their expected spatial directions (differently shaded boxes).

$$
f(p, q)=\left\|\left(v_{x}(p, q), v_{y}(p, q)\right)\right\| * \operatorname{sgn}\left(\angle\left(v_{x}(p, q), v_{y}(p, q)\right)\right), p, q=0 \ldots 127 .
$$


The next step is to quantize the estimated velocities from arbitrary real scalars to a more limited set of values. We found that direction and speed quantization significance reduces the impact of noise on the motion information around the lip area. The quantized speeds are obtained from the data by applying a mean approximation as follows.

$$
g(l, k)=\sum_{p, q=0}^{N-1} f(N l+p, N k+q), p, q=0 \ldots(N-1), l, k=0 \ldots(M-1)
$$

where $N$ and $M$ represent the window size of the boxes (Fig.1) and the number of boxes, respectively. The statistics of lip-motion are represented by 144-dimensional $(M \times M)$ feature vectors. The original dimension before reduction is $128 \times 128 \times 2=$ 32768 .

\section{Acoustic Features}

The Mel-Frequency Cepstral Coefficient (MFCC) is a commonly used instance of the filter-bank-based features [21] that can represent the speech spectrum. Here, the input signal is pre-emphasized and divided into $25-\mathrm{ms}$ frame every $10 \mathrm{~ms}$. A Hamming window is applied to each frame that is computed by (MFCC) vectors from the FFT-based, mel-warped, log-amplitude filter bank followed by a cosine transform and cepstral filtering. The speech features in this study were the MFCC vectors generated by the Hidden Markov Model Toolkit (HTK) 22] processing the data stream from the XM2VTS database. This MFCC vector contains 12 cepstral coefficients extracted from the Mel-frequency spectrum of the frame with normalized log energy, 13 delta coefficients (velocity), and 13 delta-delta coefficients (acceleration).

\section{Classification by Support Vector Machine}

The SVM formulation is based on the Structural Risk Minimization principle, which minimizes an upper bound on the generalization error, as opposed to the Empirical Risk Minimization [23] 24. An SVM is a discrimination-based binary method using a statistical algorithm. The background idea in training an SVM system is finding a hyperplane $\mathbf{w} \cdot \mathbf{x}+b=0$, as a decision boundary between two classes. For linearly separable training dataset labelled pairs $\mathbf{x}_{i}, y_{i}, i=1, \ldots, l$, where $\mathbf{x}_{i} \in \Re^{n}$ and $\mathbf{y} \in\{1,-1\}^{l}$, the following equation is verified for each observation data (feature vector).

$$
d_{i}\left(w^{T} \mathbf{x}_{i}+b\right) \geq 1-\xi_{i} \text { for } i=1,2, \ldots, l \xi_{i}>0,
$$

where $d_{i}$ is the label for sample data $\mathbf{x}_{i}$ which can be +1 or $-1 ; \mathbf{w}_{i}$ and $b$ are the weights and bias that describe the hyperplane; $\xi$ represents the number of data 
samples left inside the decision area, controlling the training errors. In our experiment we use the inner-product kernel function as RBF kernel

$$
K(\mathbf{x}, \mathbf{y})=\exp \left(-\gamma\|\mathbf{x}-\mathbf{y}\|^{2}\right), \gamma>0
$$

When conducting digit-classification experiments, we will need to choose between multiple classes. The best method of extending the two-class classifiers to multiclass problems appears to be application dependent. For our experiments we use the one against one approach. It simply constructs for each pair of classes an SVM classifier which separates those classes. All tests in this paper were performed using the SVM toolkit [25].

\section{XM2VTS Database}

All experiments in this paper are conducted on the XM2VTS database, currently the largest publicly available audio-visual database [26]. The XM2VTS database contains images and speech of 295 subjects (male and female), captured over 4 sessions. In each session, the subject is asked to pronounce three sentences when recording the video sequence; we use only "0 123456789 ". It is worth noting that the XM2VTS data is difficult to use as is for digit recognition experiments because the speech or lip motions are not annotated. Before defining a protocol we thus needed to annotate both speech and visual data, which we did nearly $100 \%$ automatically by speech segmentation. For each speaker of the XM2VTS database, the utterance "0 123456789 " was divided into single-digit sub sequences 0 to 9. We used Hidden Markov models to automatically segment the digit sequences furthermore we manually verified and corrected the segmentation results so as to eliminate the impact of database segmentation errors when interpreting our recognition results. We propose two protocol setups for the XM2VTS database; protocol 1 is the well known Lausanne protocol [26], used for speaker identification and protocol 2 which is used for digit recognition. Protocol 2 is also suggested by other studies [14.

Protocol 1 - the training contains 225 subjects with 200 subjects as clients using and 25 subjects as impostors using sessions 1, 2 and 3. The training group is also used in evaluation. For the testing session 4 of the training group are used and with yet another 70 subjects as impostors. Protocol 2 - the speakers were involved both in training SVMs and testing SVMs, we used 4 different pronunciations for training and testing. The training and test samples were completely disjoint.

\section{Experimental Results}

We want to quantify the performance of our visual features in speaker recognition and digit recognition as a stand-alone and audio-complementary modality. First the text-prompted speaker-recognition test using protocol 1 are presented and then the digit-recognition system test results using protocol 2 are presented. In our experiments we use direct fusion at feature level, which are detailed in [19]. 
Table 1. Speaker identification rate by SVM using word "7" for 295 speakers

\begin{tabular}{|l|c|c|c|}
\hline Kernel & Audio recognition rate & Visual recognition rate & Audio-Visual recognition rate \\
\hline \hline RBF & $92 \%$ & $80 \%$ & $100 \%$ \\
\hline
\end{tabular}

\subsection{Speaker-Identification System by SVM}

A smaller dataset of 100 speakers was tested for all digits and the most significant word for the speaker recognition rate was digit "7" which gave the highest recognition rate. The experiment follows protocol 1 using all 295 speakers:

- Partition the database for training, evaluation, and testing according to protocol 1.

- Train the SVM for an utterance so that the classification score, $L$ (the mean of the classification equation 6 for an utterance), is positive for the user and negative for impostors.

- $L$ is compared to a threshold $T$.

- Find the threshold $T$ such that False Acceptance is equal to False Rejection using the evaluation set.

- Using the threshold $T$, the decision $L$ is made according to the rule: if $L>$ $T$ accept the speaker else reject her/him.

However, protocol 1 is desired for verification (1:1 matching). To perform identification (1:many matching) we proceed as follows:

- Identify the speaker from a group of speakers

- We construct classifiers to separate each speaker from all other speakers in the training set.

- The speaker identity is determined by the classifier that yields the largest likelihood score.

Table 1 shows the results of using SVM classifiers with RBF kernel function using only one word (digit) to recognize the speaker identity. The recognition performance obtained when using coefficients both from dynamic image and speech are considerably higher than when using a single modality based on speech parameters. These results show that our features can perform well in identification problems.

\subsection{Digit Recognition System by SVM}

In Table2 we illustrate all systems based on only acoustic, only visual and merged audio visual feature information. We obtain the best recognition rate for digits " 1,6 , and $7 " 100 \%$. One cause why the results in Table 2 vary is that there is not enough information (especially visual information) for certain utterances. This is not surprising because the XM2VTS database was collected for identity recognition and not digit recognition. During the segmentation we could verify that when 
Table 2. Digit-recognition rate of all digits using protocol 2 in one against one SVM

\begin{tabular}{|l|c|c|c|}
\hline Word & Audio features & Visual features & Audio-Visual features \\
\hline \hline 0 & $89 \%$ & $70 \%$ & $92 \%$ \\
1 & $90 \%$ & $77 \%$ & $100 \%$ \\
2 & $86 \%$ & $60 \%$ & $89 \%$ \\
3 & $90 \%$ & $75 \%$ & $96 \%$ \\
4 & $89 \%$ & $55 \%$ & $85 \%$ \\
5 & $90 \%$ & $50 \%$ & $83 \%$ \\
6 & $100 \%$ & $90 \%$ & $100 \%$ \\
7 & $93 \%$ & $100 \%$ & $100 \%$ \\
8 & $91 \%$ & $54 \%$ & $83 \%$ \\
9 & $90 \%$ & $49 \%$ & $85 \%$ \\
\hline
\end{tabular}

uttering the words from 0 to 9 in a sequence without silence between words, the words " $4,5,8,9$ " are pronounced in shorter time-lapses and the amount of visual data is notably less in comparison to other digits. Additionally amount of speech for each speaker differ when uttering the same word or digit depending on the manner and speed of the speaker. Digit recognition give $\approx 68 \%$ and audio-visual features give $\approx 90 \%$ for overall recognition.

\section{Conclusion and Discussion}

In this paper we described a system utilizing lip movement information in dynamic image sequences of numerous speakers for robust digit and speaker recognition by no use of iterative algorithm or assuming successful lip-contour tracking. In environments such as airports, outside traffic, train station etc. the automatic digit recognition or speaker recognition system based on only acoustic information would with high probability be unsuccessful. Our experimental results support the importance of adding lip motion representation in speaker or digit recognition systems that can be installed for instance in mobile devices as a complement to acoustic information.

We presented a novel lip-motion quantization and recognition results of lipmotion features as standalone and as a complement to audio for speaker and digit recognition tasks using extensive tests. Improvements of recognition rate based on audio utilizing our motion features for digit as well as identity are provided. Our main goal is to present an effective feature level extraction for lip movement sequences which in turn can be used for identification and digit recognition as shown here and also for speaker verification [19] using an different state-of-art approach GMM.

From a visual information classification performance perspective, the digit utterance in the XM2VTS database contained less relevant information for the digits " $4,5,8,9$ ". The poor recognition performance of these digits indicate that XM2VTS database does not contain sufficient amounts of visual information on lip movements. Not surprisingly, if the visual feature-extraction is made on suffi- 
cient amount of visual speech data, the available modelling for recognition tasks appears to be sufficient for successful recognition.

\section{References}

1. Potamianos, G., Neti, C., Gravier, G., Garg, A., Senior, A.: Recent advances in the automatic recognition of audiovisual speech. Proceedings of the IEEE 91(9), 13061326 (2003)

2. Brunelli, K.R., Falavigna, D.: Person identification using multiple cues. IEEE Transactions on Pattern Analysis and Machine Intelligence 17(10), 955-966 (1995)

3. Chibelushi, C., Deravi, F., Mason, J.: A review of speech-based bimodal recognition. IEEE Transactions on Multimedia 4(1), 23-37 (2002)

4. Duc, B., Fischer, S., Bigun, J.: Face authentication with sparse grid gabor information. In: IEEE International Conference Acoustics, Speech, and Signal Processing, vol. 4(21), pp. 3053-3056 (1997)

5. Tang, X., Li, X.: Video based face recognition using multiple classifiers. In: Sixth IEEE International Conference on Automatic Face and Gesture Recognition FGR2004, pp. 345-349. IEEE Computer Society, Los Alamitos (2004)

6. Faraj, M.I., Bigun, J.: Person verification by lip-motion. In: 2006 Conference on Computer Vision and Pattern Recognition Workshop (CVPRW), pp. 37-45 (2006)

7. Luettin, J., Maitre, G.: Evaluation protocol for the extended m2vts database xm2vtsdb (1998). In: IDIAP Communication 98-054, Technical report R R-21, number $=$ IDIAP - (1998)

8. Dieckmann, U., Plankensteiner, P., Wagner, T.: Acoustic-labial speaker verification. In: Bigün, J., Borgefors, G., Chollet, G. (eds.) AVBPA 1997. LNCS, vol. 1206, pp. 301-310. Springer, Heidelberg (1997)

9. Jourlin, P., Luettin, J., Genoud, D., Wassner, H.: Acoustic-labial speaker verification. In: Bigün, J., Borgefors, G., Chollet, G. (eds.) AVBPA 1997. LNCS, vol. 1206, pp. 319-326. Springer, Heidelberg (1997)

10. Chen, T.: Audiovisual speech processing. IEEE Signal Processing Magazine 18(1), 9-21 (2001)

11. Liang, L., Zhao, X.L.Y., Pi, X., Nefian, A.: Speaker independent audio-visual continuous speech recognition. In: IEEE International Conference on Multimedia and Expo., 2002. ICME 02. Proceedings, vol. 2, pp. 26-29 (2002)

12. Kollreider, K., Fronthaler, H., Bigun, J.: Evaluating liveness by face images and the structure tensor. In: AutoID 2005: Fourth Workshop on Automatic Identification Advanced Technologies, pp. 75-80. IEEE Computer Society Press, Los Alamitos (2005)

13. Wan, V., Campbell, W.: Support vector machines for speaker verification and identification. In: Proceedings of the 2000 IEEE Signal Processing Society Workshop, Neural Networks for Signal Processing X, vol. 2, pp. 775-784 (2000)

14. Gavat, I., Costache, G., Iancu, C.: Robust speech recognizer using multiclass svm. In: 7th Seminar on Neural Network Applications in Electrical Engineering. NEUREL 2004, pp. 63-66 (2004)

15. Clarkson, P., Moreno, P.: On the use of support vector machines for phonetic classification. In: IEEE International Conference on Acoustics, Speech, and Signal Processing, ICASSP, vol. 2, pp. 585-588. IEEE Computer Society Press, Los Alamitos (1999) 
16. Reynolds, D., Quatieri, T., Dunn, R.B.: Speaker verification using adapted gaussian mixture models. Digital Signal Processing 10(1-3), 19-41 (2000)

17. Farrell, K., Mammone, R., Assaleh, K.: Speaker recognition using neural networks and conventional classifiers, vol. 2(1), pp. 194-205. IEEE-Computer Society Press, Los Alamitos (1994)

18. Bigun, J., Granlund, G., Wiklund, J.: Multidimensional orientation estimation with applications to texture analysis of optical flow. IEEE-Trans. Pattern Analysis and Machine Intelligence 13(8), 775-790 (1991)

19. Faraj, M.I., Bigun, J.: Audio-visual person authentication using lip-motion from orientation maps. (Article accepted for publication in Pattern Recognition Letters: February 2, 2007) (2007)

20. Granlund, G.H.: In search of a general picture processing operator. Computer Graphics and Image Processing 8(2), 155-173 (1978)

21. Davis, S., Mermelstein, P.: Comparison of parametric representations for monosyllabic word recognition in continuously spoken sentences. IEEE transactions on Acoustics, Speech, and Signal Processing 28(4), 357-366 (1980)

22. Young, S., Kershaw, D., Odell, J., Ollason, D., Valtchev, V., Woodland, P.: The htk book (for htk version 3.0) (2000), http://htk.eng.cam.ac.uk/docs/docs.shtml

23. Vapnik, V.N.: The Nature of Statistical Learning Theory. Springer, Heidelberg (1995)

24. Burges, C.J.: A tutorial on support vector machines for pattern recognition. Data Mining and Knowledge Discovery 2(2), 121-167 (1998)

25. Chang, C.C., Lin, C.J.: Libsvm-a library for support vector machines. software (2001), available at http://www.csie.ntu.edu.tw/cjlin/libsvm

26. Messer, K., Matas, J., Kittler, J., Luettin, J.: Xm2vtsdb: The extended m2vts database. In: Second International Conference of Audio and Video-based Biometric Person Authentication, ICSLP'96, pp. 72-77 (1999) 